\title{
Repeated flu shots may blunt effectiveness
}

U niversal influenza immunization programs, available in virtually every province and territory, may need to be reconsidered in light of emerging evidence that repeated flu shots may blunt the vaccine's effectiveness in subsequent seasons.

That phenomenon was seen in the Jan. 29, 2015 interim estimates of the effectiveness of the 2014/15 vaccine against influenza A (H3N2) from Canada's Sentinel Physician Surveillance Network, headed by Dr. Danuta Skowronski of the British Columbia Centre for Disease Control.

The effects of repeat immunizations need to be studied further. Meanwhile, a return to targeted, high-risk flu vaccine programs, rather than universal coverage, seems warranted, said Skowronski, the BC centre's epidemiology lead for influenza and emerging respiratory pathogens.

At the same time, antivirals - formerly plan B in the primary prevention of influenza - should be used early. "There's a lot of debate about antivirals and that needs to be resolved also, but for now, [early antiviral use] should come to the fore," Skowronski said.

The BC network's estimates of this year's flu vaccine efficacy, published in Eurosurveillance, were $-8 \%$ overall and $2 \%$ in young adults against medically attended, laboratory-confirmed influenza A (H3N2) infection - which Skowronski said she interprets as a null effect. This also represents the lowest measured protection against a seasonal virus in the program's 10-year history, she added.

It recently became known that this year's $\mathrm{H} 3 \mathrm{~N} 2$ virus was not a match for the H3N2 component of this year's trivalent vaccine, but the authors of this new study also saw variability in vaccine effectiveness that was related to prior vaccination history. Vaccine effectiveness was $43 \%$ for those who hadn't received the 2013/14 vaccine but $-15 \%$

for participants who received both seasons' vaccines.

Those are not statistically significant findings, "but when you're dealing with a low vaccine effectiveness, even $40 \%$, and a null effect in those who received vaccine the previous year, you need a

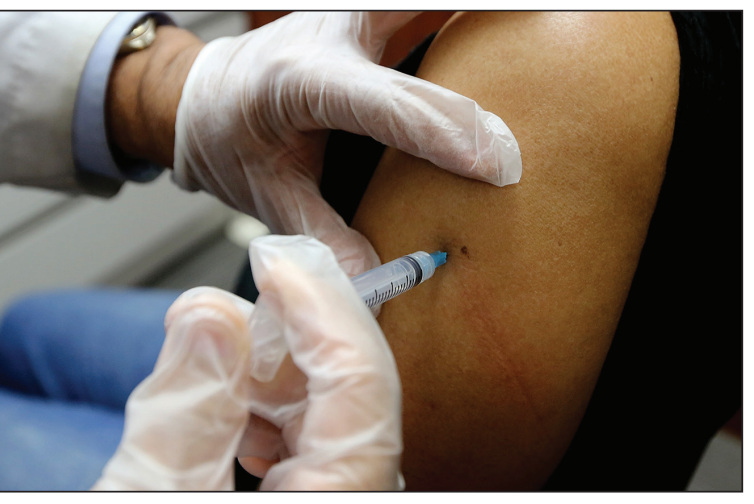

Research in both Canada and the US indicates that getting the flu hot several years in a row may lessen the vaccine's effectiveness.

vaccine effectiveness in those who'd had flu vaccine over the previous five years, compared to those who'd had only the previous year's vaccine or no vaccine previously.

The findings are also consistent with a 1999 observation of variable flu vaccine efficacy depending on immunization history, by Derek J. Smith, the current director, WHO Collaborating Centre for Modelling, Evolution and Control of Emerging Infectious Diseases. Smith proposes "antigenic distance" as an explanation. This hypothesis holds that the impact on the current season's vaccine effectiveness is most pronounced when the difference between last season's vaccine component and the current season's vaccine component is small

massive sample size to prove that statistically," Skowronski said. "If we wait for that we'll never be able to tease apart these signals."

Dr. Richard Schabas, Ontario's former chief medical officer who is now medical officer of health in the Hastings and Prince Edward Counties Health Unit in Belleville, Ontario, agrees that it's time to revisit universal flu vaccine programs, which are now available in all Canadian provinces and territories except BC, Quebec and New Brunswick.

He was once a proponent of universal programs but now says, "We should use the next six months or so to do a very careful rethink of where we are. There's enough new evidence that we should all be troubled enough by, that we should be taking a long and sober look at our policies. There are more and more unanswered questions about how effective a universal program really is."

Canada's Sentinel Physician Surveillance Network has previously observed this decrease in effectiveness, as have others. In the September 2014 issue of Clinical Infectious Diseases, US investigators documented a lower (this year they're identical).

In that case, the current season's vaccine may boost memory of the earlier vaccine's antibody responses; then these "old" antibodies may mop up the current vaccine's antigen before the newly vaccinated person can mount an immune response to it (this is called antibody interference).

For now, researchers need to investigate the phenomenon, Skowronski said. And public health officials need to reconsider the wisdom of targeted flu vaccine programs, especially in a year when the elderly have been disproportionately affected.

On Feb. 5, 2015, other Canadian researchers published interim estimates of 2014/15 influenza vaccine effectiveness in preventing laboratoryconfirmed influenza-related hospitalization. The figure for people aged 65 and over was $-32.9 \%$, according to findings from the Serious Outcomes Surveillance Network of the Canadian Immunization Research Network. Terry Murray, Toronto, Ont.

CMAJ 2015. DOI:10.1503/cmaj.109-5000 